Document downloaded from:

http://hdl.handle.net/10251/43470

This paper must be cited as:

Ballester-Bolinches, A.; Beidleman, J.; Esteban Romero, R.; Pérez-Calabuig, V. (2013). Maximal subgroups and PST-groups. Central European Journal of Mathematics. 11(6):1078-1082. doi:10.2478/s11533-013-0222-z.

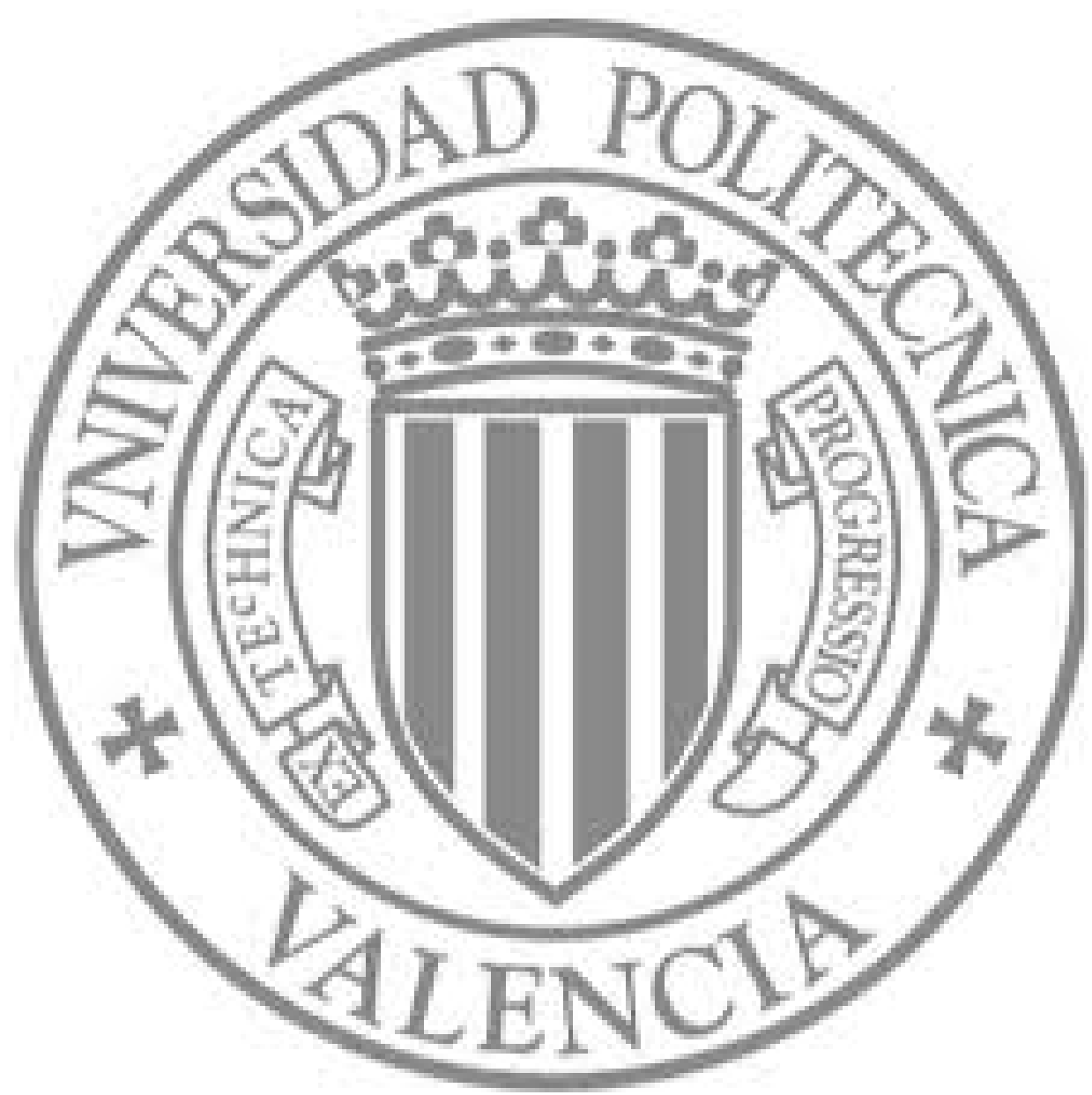

The final publication is available at

http://link.springer.com/article/10.2478\%2Fs11533-013-0222-z

Copyright Springer Verlag (Germany) 


\title{
Maximal subgroups and PST-groups
}

\author{
A. Ballester-Bolinches* ${ }^{*}$ J. C. Beidleman ${ }^{\dagger}$ \\ R. Esteban-Romero ${ }^{\ddagger} \quad$ V. Pérez-Calabuig ${ }^{\S}$
}

\begin{abstract}
A subgroup $H$ of a group $G$ is said to permute with a subgroup $K$ of $G$ if $H K$ is a subgroup of $G$. $H$ is said to be permutable (resp. $S$-permutable) if it permutes with all the subgroups (resp. Sylow subgroups) of $G$. Finite groups in which permutability (resp. $S$-permutability) is a transitive relation are called PT-groups (resp. PST-groups). PT-, PST- and T-groups, or groups in which normality is transitive, have been extensively studied and characterised. Kaplan (Arch. Math., 96 (2011), 19-25) presented some new characterisations of soluble Tgroups. The main goal of this paper is to establish PT- and PSTversions of Kaplan's results, which enables a better understanding of the relationships between these classes.

Mathematics Subject Classification (2010): 20D05, 20D10, 20E15, 20E28, $20 \mathrm{~F} 16$

Keywords: finite groups, permutability, Sylow-permutability, maximal subgroups, supersolubility.
\end{abstract}

\section{Introduction and statement of results}

All groups considered in this paper are finite.

Recall that a subgroup $A$ of a group $G$ is said to be permutable (resp. $S$-permutable) in $G$ if $A$ permutes with all the subgroups (resp. Sylow subgroups) of $G$. Kegel $([1,1.2 .14(3)])$ showed that any $S$-permutable subgroup

*Departament d'Àlgebra, Universitat de València; Dr. Moliner, 50; 46100, Burjassot, València, Spain; email: Adolfo.Ballester@uv.es

${ }^{\dagger}$ Department of Mathematics, University of Kentucky, Lexington, KY 40506-0027, USA, email: james.beidleman@uky.edu

¥Institut Universitari de Matemàtica Pura i Aplicada, Universitat Politècnica de València; Camí de Vera, s/n; 46022 València, Spain, email: resteban@mat.upv.es

${ }^{\S}$ Departament d'Àlgebra, Universitat de València; Dr. Moliner, 50; 46100, Burjassot, València, Spain; email: vipeca3@alumni.uv.es 
is subnormal. A group $G$ is called a $P T$-group (resp. PST-group) if permutability (resp. $S$-permutability) is a transitive relation in $G$, that is, if $A$ and $B$ are subgroups of $G$ such that $A$ is permutable (resp. $S$-permutable) in $B$ and $B$ is permutable (resp. $S$-permutable) in $G$, then $A$ is permutable (resp. $S$-permutable) in $G$. By Kegel's result $G$ is a PT-group (resp. PST-group) if and only if every subnormal subgroup is permutable (resp. $S$-permutable) in $G$. Clearly every PT-group is a PST-group and every T-group, or group in which every subnormal subgroup is normal, is a PT-group. The reader is referred to [1, Chap. 2] for basic results about these classes of groups.

The results presented here spring from recent results of Kaplan [6] characterising the soluble T-groups by means of their maximal subgroups and some class of prefrattini subgroups. He proved that a group $G$ is a soluble T-group if and only if every non-normal subgroup of every subgroup $H$ of $G$ is contained in a non-normal maximal subgroup of $H$. He also considers the intersection of all maximal subgroups of a group $G$ containing a proper subgroup $A$ of $G$ to give a criterion for a soluble group to be a T-group.

This paper is a natural continuation of Kaplan's paper [6] and presents new characterisations of soluble PT- and PST-groups. Kaplan's results follow naturally from our main theorems. It is abundantly clear that our results are not a mere exercise in generalisation. In fact, our theorems shed more light on the study of relations between these three classes in the soluble universe and open the door to possible extensions to the non-soluble one.

The starting point of our results is the following: let $A$ be a proper permutable (resp. $S$-permutable) subgroup of a soluble group $G$. Then, by Kegel's result, $A$ is subnormal in $G$ and so it is contained in a maximal subgroup of $G$ which is normal in $G$. It then seems desirable to investigate the class of groups in which every non-permutable (resp. non- $S$-permutable subgroup) is contained in a non-normal maximal subgroup.

Definition 1.1. We say that a group $G$ is a PNM-group (resp. SNM-group) if every non-permutable (resp. non- $S$-permutable) subgroup of $G$ is contained in a non-normal maximal subgroup of $G$.

It is clear that every PNM-group is an SNM-group. If $G$ has all subgroups $S$-permutable, or equivalently, $G$ is nilpotent, then $G$ is an SNM-group. However, a nilpotent group $G$ is a PNM-group if and only if every subgroup of $G$ is permutable, that is, $G$ is an Iwasawa group. If $G$ is a perfect group, then every maximal subgroup is non-normal, and so $G$ is also a PNM-group. The classes of all PNM-groups and SNM-groups are not closed under taking subgroups since the symmetric group of degree four is a PNM-group but the alternating group of degree four is a non-PNM-group. However, both classes are closed under taking epimorphic images. 
Our first main result is the following:

Theorem 1.2. A group $G$ is a soluble PST-group if and only if every subgroup of $G$ is an SNM-group.

Lemma 1.3. Every subgroup of a group $G$ is a PNM-group if and only if every subgroup of $G$ is an SNM-group and the Sylow subgroups of $G$ are Iwasawa groups.

Applying Theorem 1.2 and Lemma 1.3, we have:

Corollary 1.4. A group $G$ is a soluble PT-group if and only if every subgroup of $G$ is a PNM-group.

Following [6], we say that a group $G$ is an NNM-group if every non-normal subgroup is contained in a non-normal maximal subgroup. It is clear that a nilpotent group is an NNM-group if and only if it is a Dedekind group. Hence, applying [1, 2.1.10], we see that every subgroup of a group $G$ is an NNM-group if and only if every subgroup of $G$ is an SNM-group and the Sylow subgroups are Dedekind. As an immediate deduction we have the

Corollary 1.5 ([6, Theorem 1]). A group $G$ is a soluble T-group if and only if every subgroup is an NNM-group.

Our next result extends [6, Theorem 5]. It shows that all the SNMextensions of a supersoluble group by a nilpotent group are supersoluble.

Theorem 1.6. If $G$ is an SNM-group, then the nilpotent residual $G^{\mathfrak{N}}$ is supersoluble if and only if $G$ is supersoluble.

Recall that the nilpotent residual of a group is the smallest normal subgroup with nilpotent quotient.

Kaplan [6, Theorem 7] considered the class of all groups in which every non-nilpotent subgroup is an NNM-group. He proved this class is composed of supersoluble groups. Let $G$ be a group such that every non-nilpotent subgroup of $G$ belongs to SNM. If $G$ is not nilpotent, then $G$ is an SNM-group. Moreover, every subgroup of $G^{\mathfrak{N}}$ is an SNM-group since every nilpotent group belongs to SNM. Applying Theorem 1.2, we obtain that $G^{\mathfrak{N}}$ is a soluble PSTgroup and so $G^{\mathfrak{N}}$ is supersoluble by $[1,2.1 .2]$. Consequently, $G$ is supersoluble by Theorem 1.6. We have proved:

Corollary 1.7. If $G$ is a group whose non-nilpotent subgroups are SNMgroups, then $G$ is supersoluble. 
Let $A$ be a proper subgroup of $G$. Following [6], we denote by $\Lambda_{G}(A)$ the intersection of all maximal subgroups of $G$ containing $A$. It is clear that $\Lambda_{G}\left(\Lambda_{G}(A)\right)=\Lambda_{G}(A)$. If $N$ is a normal subgroup of $G$ contained in $\Lambda_{G}(A)$, then $\Lambda_{G}(A)=\Lambda_{G}(A N)$, and $\Lambda_{G / N}(A N / N)=\Lambda_{G}(A) / N$. If $A$ is normal in $G$, then $\Lambda_{G}(A)$ is the intersection of conjugacy classes of maximal subgroups of $G$, and therefore $\Lambda_{G}(A)$ is normal in $G$. According to [6, 2.1], if $A$ is subnormal in $G$, then $\Lambda_{G}(A)=\Lambda_{G}\left(A^{G}\right)$. In particular, $\Lambda_{G}(A)$ is normal in $G$. As a consequence, $\Lambda_{G}(A)$ is subnormal in $G$ if and only if $\Lambda_{G}(A)$ is normal in $G$.

Kaplan [6] considered the class of all groups $G$ in which the set of all non-normal subgroups is $\Lambda_{G}$-invariant, and he proved that this class is, in the soluble universe, the class of all T-groups. Bearing in mind this result, it seems natural to wonder how nearly a group $G$ in which the set of all non-permutable (resp. $S$-permutable) subgroups of $G$ is $\Lambda_{G}$-invariant is a PT-group (resp. PST-group).

Assume that $G$ is a group such that $\Lambda_{G}(A)$ is not $S$-permutable for all non- $S$-permutable subgroups $A$ of $G$. Then if $A$ is subnormal in $G$, then $\Lambda_{G}(A)$ is normal in $G$ by the above observations. Therefore $A$ must be $S$ permutable in $G$, and the same is true for permutable subgroups. Our next results show that the converse is also true.

Theorem 1.8. Let $G$ be a soluble PST-group. If $A$ is a subgroup of $G$ such that $\Lambda_{G}(A)$ is normal in $G$, then $A$ is $S$-permutable in $G$.

Theorem 1.8 will now be applied to the consideration of soluble PSTgroups.

Corollary 1.9. A soluble group $G$ is a PST-group if and only if the set of all non-S-permutable subgroups of $G$ is $\Lambda_{G}$-invariant.

Applying [1, 2.1.10], we have that $S$-permutable subgroups of groups with Dedekind (resp. Iwasawa) Sylow subgroups are normal (resp. permutable). Hence [6, Theorem 2] is a consequence of the above corollary. In fact, we have:

Corollary 1.10. A soluble group $G$ is a T-group (resp. PT-group) if and only if the set of all non-normal (resp. non-permutable) subgroups of $G$ is $\Lambda_{G}$-invariant.

\section{Proofs of the results}

Proof of Theorem 1.2. Let $G$ be a soluble PST-group, and let $L=G^{\mathfrak{N}}$ be the nilpotent residual of $G$ and $D$ a system normaliser of $G$. By [1, 2.1.8], 
$L$ is an abelian Hall subgroup upon which $D$ acts by conjugation as power automorphisms. Moreover, $D$ is a complement of $L$ in $G$ by $[2,4.2 .17]$. We prove that $G$ is an SNM-group by induction on the order of $G$. Let $A$ be a non- $S$-permutable subgroup of $G$. Then $1 \neq L$, and $A /(A \cap L)$ is a non$S$-permutable subgroup of $G /(A \cap L)$. Now, $A \cap L=1$, for otherwise, by induction, $A /(A \cap L)$ would be contained in a non-normal maximal subgroup $M /(A \cap L)$ of $G /(A \cap L)$. Then $M$ would be a non-normal maximal subgroup of $G$ containing $A$. Hence $A \cap L=1$. Since $L$ and $D$ are Hall subgroups of $G$, we may assume that $A$ is contained in $D$. Let $M$ be a maximal subgroup of $G$ containing $D$. Then, by [2, 4.1.12], $M$ is not normal in $G$. Consequently, $G$ is an SNM-group. Now, applying [1, 2.1.9], every subgroup of $G$ is a soluble PST-group. The above argument implies that every subgroup of $G$ is an SNM-group.

Now it will be established that every group $G$ in which every subgroup is an SNM-group must be a PST-group. We argue by contradiction. Assume that $G$ is a counterexample of least order to the result. Then every proper subgroup of $G$ is a soluble PST-group and so every proper subgroup of $G$ is supersoluble by [1, 2.1.2]. Applying [5, Satz 22], we conclude that $G$ is soluble.

On the other hand, there exists a subnormal subgroup $H$ which is not $S$-permutable in $G$. Let $M$ be a maximal normal subgroup of $G$ containing $H$. As $G$ is an SNM-group, we can find a non-normal maximal subgroup $L$ of $G$ containing $H$. Then $G=M L$. Since $H$ is not $S$-permutable in $G$, there exists a Sylow $p$-subgroup $P$ of $G$ such that $P$ does not permute with $H$. The minimal choice of $G$ implies that $H$ is $S$-permutable in $M$ and $L$. By $[1,1.1 .19]$ there exists a Sylow $p$-subgroup $A$ of $M$ and a Sylow $p$-subgroup $B$ of $L$ such that their product $Q=A B$ is a Sylow $p$-subgroup of $G$. Let $x \in G$ such that $P^{x}=Q$. Then $H$ permutes with both $A$ and $B$, so $H$ permutes with $Q$. Consider a minimal normal subgroup $N$ of $G$ contained in $M$. Since the properties of $G$, as enunciated in the statement of the theorem, are inherited by quotients of $G$, the minimality of $G$ implies that $H N$ permutes with $P$. If $(H N) P$ were a proper subgroup of $G$, then $H$ would permute with $P$. This would contradict our choice of $G$. Therefore $G=P(H N)$, and $x=a b$ with $a \in P$ and $b \in H N$. By [3, A, 14.3], $H$ is a normal subgroup of $H N$. Since $H$ permutes with $P^{b}$, it follows that $H^{b^{-1}}=H$ permutes with $P$, contrary to assumption.

Proof of Lemma 1.3. Assume that every subgroup of a group $G$ is a PNMgroup. Then every subgroup of $G$ is an SNM-group since permutability implies $S$-permutability. Moreover, every Sylow subgroup $P$ of $G$ is a nilpotent PNM-group. Hence $P$ has all its subgroups permutable and so $P$ is an 
Iwasawa group.

Conversely assume that every subgroup of $G$ is an SNM-group and the Sylow subgroups of $G$ are Iwasawa groups. Let $K$ be an $S$-permutable subgroup of a subgroup $H$ of $G$. Since the Sylow subgroups of $H$ are also Iwasawa groups, we can apply $[1,2.1 .10]$ to conclude that $K$ is permutable in $H$. Therefore $H$ is a PNM-group. Consequently every subgroup of $G$ is a PNM-group.

Proof of Corollary 1.4. If $G$ is a soluble PT-group, then $G$ is a PST-group whose Sylow subgroups are Iwasawa by [1, 2.1.12]. Moreover, every subgroup of $G$ is an SNM-group by Theorem 1.2. Therefore Lemma 1.3 implies that every subgroup of $G$ is a PNM-group.

Suppose now that every subgroup of $G$ is a PNM-group. Then, by Lemma 1.3, every subgroup of $G$ is an SNM-group and the Sylow subgroups of $G$ are Iwasawa groups. By virtue of Theorem 1.2, $G$ is a soluble PST-group. Now we can apply $[1,2.1 .12]$ to conclude that $G$ is a soluble PT-group.

Proof of Theorem 1.6. It is clear that only the necessity of the condition is in doubt. We use induction on the order of $G$. Note that $G$ is soluble, and we may assume $G^{\mathfrak{N}} \neq 1$. Let $N$ be a minimal normal subgroup of $G$. Then $G^{\mathfrak{N}} N / N$ is the nilpotent residual of $G / N$ by $[2,2.2 .8(\mathrm{a})]$. Moreover, $G^{\mathfrak{N}} N / N$ is supersoluble and $G / N$ is an SNM-group. By induction, $G / N$ is supersoluble. Since the class of all supersoluble groups is a saturated formation, we may suppose that $G$ is a primitive group, that is, $G$ has a unique minimal normal subgroup, $N$ say, $C_{G}(N)=N$, and $N$ is complemented in $G$ by a core-free maximal subgroup $M$ of $G$ (see $[2,1.1 .7]$ ). Clearly, $N$ is contained in $G^{\mathfrak{N}}$. Let $p$ be the prime dividing $|N|$. Then $N$ has the structure of an irreducible module for $M$ over the field of $p$ elements in a natural way. Applying $[2,2.2 .8(2)], M^{\mathfrak{N}} N=G^{\mathfrak{N}}$. Clifford's theorem [3, B, 7.3] implies the complete reducibility of $N$ as a direct product of minimal normal subgroups of $G^{\mathfrak{N}}$. Let $A$ be minimal normal subgroup of $G^{\mathfrak{N}}$ contained in $N$. Then $A$ has order $p$ as $G^{\mathfrak{N}}$ is supersoluble. If $A M^{\mathfrak{N}}$ were not $S$-permutable in $G$, there would exist a non-normal maximal subgroup $L$ of $G$ containing $A M^{\mathfrak{N}}$. Since the core-free maximal subgroups of $G$ are conjugate by $[2,1.1 .10]$ and $A \leq(L \cap N)$, it follows that $N$ is contained in $L$. In particular, $G^{\mathfrak{N}}$ is contained in $L$ and $L$ is normal in $G$. This contradiction shows that $A M^{\mathfrak{N}}$ is $S$-permutable in $G$. Applying $[1,1.2 .14(3)]$, we obtain that $A M^{\mathfrak{N}}$ is subnormal in $G$ and so $N$ normalises $A M^{\mathfrak{N}}$ by $[3, \mathrm{~A}, 14.3]$. Therefore $\left[M^{\mathfrak{N}}, N\right] \leq\left(A M^{\mathfrak{N}}\right) \cap N=A$ and this holds for every minimal normal subgroup of $G^{\mathfrak{N}}$ contained in $N$. If $A=N$, then $N$ is of prime order and $G$ is supersoluble. Hence $N$ is a direct product of at least two minimal normal subgroups of $G^{\mathfrak{N}}$. In this case, 
$M^{\mathfrak{N}}$ centralises $N$ and so $M^{\mathfrak{N}}=1$. In this case, every subgroup of $N$ is $S$-permutable in $G$. By [1, 2.1.3], it follows that $N$ is of prime order and hence $G$ is supersoluble. This establishes the theorem.

Proof of Theorem 1.8. Suppose the result false, and let the PST-group $G$ provide a counterexample of least possible order. Then $G$ has a non- $S$ permutable subgroup $A$ of $G$ such that $\Lambda_{G}(A)$ is normal in $G$. Let $N$ be a minimal normal subgroup of $G$ contained in $\Lambda_{G}(A)$. Then $\Lambda_{G}(A) / N=$ $\Lambda_{G / N}(A N / N)$ is normal in $G / N$. The minimal choice of $G$ implies that $A N / N$ is $S$-permutable in $G / N$. Hence $A N$ is $S$-permutable in $G$ and so Core $_{G}(A)=1$. Let $L$ be the nilpotent residual of $G$ and let $C$ be a system normaliser of $G$ complementing $L$. Since each subgroup of $L$ is normal in $G$ by $[1,2.1 .8]$, it follows that $A \cap L=1$. Since $L$ and $C$ are Hall subgroups of $G$, we may conclude that $A$ is contained in $C$. Then $\Lambda_{G}(A) \leq \Lambda_{G}(C)$. Let $M$ be a non-normal maximal subgroup of $G$. Applying [2, 4.1.13] we have that $M$ contains a conjugate of $C$. Hence $M$ contains a conjugate of $\Lambda_{G}(C)$ and therefore $\Lambda_{G}(A)$ is contained in $M$. This means that $\Lambda_{G}(A)$ is contained in the intersection $L(G)$ of all non-normal maximal subgroups of $G$. Applying [4], $L(G)$ is nilpotent. Consequently, $\Lambda_{G}(A)$ is contained in the Fitting subgroup of $G$ and so $A$ is subnormal in $G$. Since $G$ is a PSTgroup, it follows that $A$ is $S$-permutable in $G$. This contradiction allows us to conclude that the result as stated is true.

\section{Acknowledgements}

The first and third author have been supported by the research grant MTM201019938-C03-01 from MICINN (Spain).

\section{References}

[1] A. Ballester-Bolinches, R. Esteban-Romero, and M. Asaad. Products of finite groups, volume 53 of de Gruyter Expositions in Mathematics. Walter de Gruyter, Berlin, 2010.

[2] A. Ballester-Bolinches and L. M. Ezquerro. Classes of Finite Groups, volume 584 of Mathematics and its Applications. Springer, New York, 2006.

[3] K. Doerk and T. Hawkes. Finite Soluble Groups, volume 4 of De Gruyter Expositions in Mathematics. Walter de Gruyter, Berlin, New York, 1992. 
[4] W. Gaschütz. Über die $\Phi$-Untergruppe endlicher Gruppen. Math. Z., 58:160-170, 1953.

[5] B. Huppert. Endliche Gruppen I, volume 134 of Grund. Math. Wiss. Springer Verlag, Berlin, Heidelberg, New York, 1967.

[6] Gil Kaplan. On T-groups, supersolvable groups, and maximal subgroups. Arch. Math. (Basel), 96(1):19-25, 2011. 